\title{
Comparison of Methods for Calculating Coupling Length in Step-Index Optical Fibers
}

\author{
S. SavoviĆ $\bar{c}^{a, b, *}$, A. Djordjevich ${ }^{b}$, B. DrluaČA ${ }^{a}$ And M.S. KovaČeviĆ ${ }^{a}$ \\ ${ }^{a}$ Faculty of Science, R. Domanovica 12, 34000 Kragujevac, Serbia \\ ${ }^{b}$ City University of Hong Kong, 83 Tat Chee Avenue, Hong Kong, China
}

\begin{abstract}
A recently reported function for calculation of the coupling length at which the equilibrium mode distribution is achieved in step-index plastic optical fibers is compared to a long established calculation method and to experimental findings. The recent function, while simpler to apply as it eliminates the need to numerically solve the power flow equation repeatedly for every case, is also more accurate for high numerical aperture $(0.45$ to 0.5$)$ plastic optical fibers.
\end{abstract}

PACS numbers: 42.81.-i, 42.81.Qb, 42.81.Uv

\section{Introduction}

Mode coupling in a step-index (SI) plastic optical fiber transfers power between neighboring modes. It thus progressively alters the input power distribution along the fiber length. Consequently, it has been described as strongly affecting transmission characteristics of the fiber [1-5]. Mode coupling is caused by fiber impurities and inhomogeneities introduced during the fiber manufacturing process. Examples of such imperfections are microscopic bends, diameter variations, irregularity of the core-cladding boundary, and refractive index distribution fluctuations [6].

Due to mode coupling, the optical power distribution at the output end of the fiber depends not only on the launch conditions, but also on fiber properties and length. For example, light launched along a cone with a specific angle relative to the fiber axis will form a sharply defined ring radiation pattern at the output end of only short fibers. Because of mode coupling, the boundary (edges) of such a ring become blurred or fuzzy at the end of longer fibers. The extent of this fussiness increases with fiber length and the ring-pattern evolves gradually into a disk. The disk extends across the entire fiber core when the "coupling length" $L_{\mathrm{c}}$ of the fiber is reached.

An equilibrium mode distribution (EMD) exists beyond the coupling length $L_{\mathrm{c}}$ of the fiber. It is characterized by the absence of rings regardless of launch conditions (i.e. angle of launch). While extending fully across the fiber core, the resulting disk pattern is not yet uniform throughout as light distribution across it still varies with launch conditions. Nevertheless, EMD indicates a substantially complete mode coupling. It is of critical importance when measuring characteristics of multimode

\footnotetext{
* corresponding author; e-mail: savovic@kg.ac.rs
}

optical fibers (linear attenuation, band width, etc.). Measurement of these characteristics is considered meaningful only if performed at or beyond the EMD condition when it is possible to assign to a fiber a unique value of loss per unit length [1]. At distance $z_{\mathrm{s}}\left(z_{\mathrm{s}}>L_{\mathrm{c}}\right)$ from the input fiber end, all individual disk patterns corresponding to different launch angles take the same light-distribution across the fiber core. The "steady state distribution" (SSD) is then said to have been achieved as the output light distribution becomes independent of launch conditions. SSD indicates the full completion of (the effects of) the mode coupling process (actually the coupling process continues but with no further apparent effects).

To experimentally determine the fiber length $L_{\mathrm{c}}$ where the EMD is achieved, pulse broadening measurements can be performed for different fiber lengths $z$ while trying to identify the length $z=L_{\mathrm{c}}$ beyond which the band width becomes proportional to $1 / z^{1 / 2}$ instead of $1 / z$ $[2,7]$. An alternative method of determining $L_{\mathrm{c}}$ is to identify the fiber length after which all output angular power distributions take the disk form regardless of the incidence angle (within the numerical aperture (NA) of the fiber).

As for analytical methods, most reported calculations make use of the coupling constant $D$ in Gloge's power flow equation [8], which is a partial differential equation. In this paper, the coupling length $L_{\mathrm{c}}$ for achieving EMD in SI plastic optical fiber (POF) is calculated also using a recently reported function [9]. This allowed the comparison of the two methods with respect to reported experimental findings.

\section{Calculation of the coupling length $L_{\mathrm{c}}$}

Assuming that mode coupling in multimode optical fibers occurs predominantly between adjacent modes, 
Gloge has derived his time-dependent power flow equation (1) [7]. It determines the power distribution in the core $P(\theta, z, t)$ as a function of the propagation angle $\theta$ (measured relative to the core axis), coordinate along the fiber axis $z$ (measured from the input fiber end), and time $t$. This power distribution $P(\theta, z, t)$ is related to the modal attenuation, modal dispersion, and mode coupling, which are represented by three respective terms on the right side of $(1)$ :

$$
\begin{aligned}
& \frac{\partial P(\theta, z, t)}{\partial z}=-A \theta^{2} P(\theta, z, t)-\frac{n}{2 c} \theta^{2} \frac{\partial P(\theta, z, t)}{\partial t} \\
& +\frac{D}{\theta} \frac{\partial}{\partial \theta}\left(\theta \frac{\partial P(\theta, z, t)}{\partial \theta}\right),
\end{aligned}
$$

where $A$ is the second-order multiplicative factor in the series expansion of the power loss coefficient $\alpha(\theta)$ due to absorption and scattering: $\alpha(\theta)=\alpha_{0}+A \theta^{2}+\ldots$; $c$ is the velocity of light in vacuum; and $D=d_{0}(\lambda / 4 a n)^{2}$ in which $\lambda, a$ and $n$ are the free-space wavelength of light, core radius and refractive index of the core, respectively, while $d_{0}$ is the zero-order term in the series expansion of the expression for the coupling coefficient $d(\theta)=d_{0}+\alpha(\theta) \theta^{2}+\ldots$ The tacit assumption that the coupling coefficient $d(\theta)$ is constant (equal to $d_{0}$ ) has been used routinely for SI POF in the absence of reliable estimates of other terms in the expansion series of $d(\theta)$ [6, 8-14].

Using the power-flow Eq. (1), Gloge obtained that the broadening of the transmitted (relative to the input) pulse width in step-index optical fibers progresses slower with, than without, mode coupling [7]. This broadening in the presence of coupling effects is proportional to $z^{1 / 2}$ (rather than $z$ without coupling):

$$
\tau=\frac{n^{2} \sin ^{2}\left(\Theta_{\infty}\right)}{2 n c}\left(\frac{z}{4 \gamma_{\infty}}\right)^{1 / 2},
$$

where $\Theta_{\infty}=(4 D / A)^{1 / 4}$ is the angular width of the steady state mode distribution and $\gamma_{\infty}=(4 D A)^{1 / 2}$ is the overall loss coefficient.

Expression (2) reveals the improvement and penalty resulting from mode coupling. On the one hand, uncoupled and uniformly attenuated modes cause the impulse response to broaden to a higher effective width of $z\left(n \sin \Theta_{\infty}\right)^{2} / 2 n c$ in $z \mathrm{~km}$ of fiber. Mode coupling reduces this undesirable effect by a factor of $\left(4 \gamma_{\infty} z\right)^{1 / 2}$. On the other hand, the overall attenuation increases by $4.35 \gamma_{\infty} \mathrm{dB} / \mathrm{km}$ in the presence of coupling. For tradeoff analysis, "coupling length" $L_{\mathrm{c}}$ is defined as:

$$
L_{\mathrm{c}}=\frac{1}{4 \gamma_{\infty}}=\frac{1}{8(D A)^{1 / 2}} .
$$

This coupling length marks the point at which the width of the impulse response changes from linear to a square-root dependence of length.

In our previous works we have shown that coupling length of SI POFs depends both on NA of the fiber [13] as well as on the width of the launch beam distribution [9]. One can calculate the coupling length in SI POFs with high NA (0.45 to 0.5$)$ using equation [9]: i.e.

$$
\log L_{\mathrm{c}}=a+b \log D
$$

$$
L_{\mathrm{c}}=10^{a} \mathrm{D}^{b},
$$

where $a=-1.9700 \pm 0.0448$ and $b=-1.0243 \pm 0.0168$ for laser launch beam distributions (full width at half maximum (FWHM) of $2.5^{\circ}$ was assumed); and $a=$ $-2.2908 \pm 0.0069$ and $b=-0.9986 \pm 0.0026$ for LED launch beam distributions (FWHM of $33^{\circ}$ was assumed).

The expression (4) takes two different sets of coefficients for different launch-beam widths. This contrasts Gloge's result (3) in which the coupling length is independent of the width of the launch beam distribution. We will verify the accuracy of the proposed function (4) for calculating the coupling length $L_{\mathrm{c}}$.

\section{Results}

In order to test and compare the Gloge function (3) and our recently proposed function (4), we used them to evaluate the coupling length $L_{\mathrm{c}}$ for high-NA SI POF investigated experimentally by Zubia et al. [11]. This fiber's core diameter is $d=0.98 \mathrm{~mm}$. Its refractive index of the core is $n=1.492$, theoretical numerical aperture $\mathrm{NA}=0.5$, and critical angle $\theta_{\mathrm{c}}=19.6^{\circ}$ (measured inside the fiber), i.e. $\theta_{c}=30^{\circ}$ (measured in air). Values of $D=7.5 \times 10^{-4} \mathrm{rad}^{2} / \mathrm{m}$ and $A=1.37\left(\mathrm{rad}^{2} \mathrm{~m}\right)^{-1}$ have been reported for this fiber [11]. Using Gloge's Eq. (3), we obtain coupling length $L_{\mathrm{c}}=3.9 \mathrm{~m}$. Using Eq. (4), we obtain $L_{\mathrm{c}}=17 \mathrm{~m}$, where $a=-1.9700 \pm 0.0448$ and $b=-1.0243 \pm 0.0168$ for laser launch beam distributions with FWHM of $2.5^{\circ}$, since Zubia et al. have used laser source in their experiment. In our previous work [14], by solving the time-independent power flow equation (Eq. (1) without the second term on the right-hand side), we have obtained that $L_{\mathrm{c}}=17.6 \mathrm{~m}$. Zubia et al.'s experimental results [11] showed for $3 \mathrm{~m}$ long optical fiber that the coupling of only lower order modes occurs at such short fiber length while higher order modes have apparently still not started to couple. This is experimental evidence that the coupling length $L_{\mathrm{c}}=3.9 \mathrm{~m}$ obtained using Gloge's function is not accurate and that a longer fiber length $\left(L_{\mathrm{c}}=17.6 \mathrm{~m}\right)$ is needed for achieving EMD.

A similar value for $L_{\mathrm{c}}=15-20 \mathrm{~m}$ has been obtained experimentally by Garito et al. [6] for high NA (0.51) SI POF. Short coupling lengths of 0.5 to $4 \mathrm{~m}$ for laser launch beam input were reported only for SI POF's strained by many small-radius bends $[15,16]$. In strained fibers due to their tight bends introduced an additional coupling effect (by curvature) occurs that is reflected in a more than one order of magnitude higher coupling coefficient $D$ for the overall fiber configuration (of $D \approx 10^{-3}$ to $10^{-2} \mathrm{rad}^{2} / \mathrm{m}$ depending on the number, frequency and severity of bends in the configuration; whereas for unstrained fibers $D \approx 10^{-4} \mathrm{rad}^{2} / \mathrm{m}$ and $D=5.62 \times 10^{-4}$ $\mathrm{rad}^{2} / \mathrm{m}$ in Garito's case [6]). As a consequence, equilibrium mode distribution in strained fibers is achieved at shorter distances than for straight fibers. 
It follows that our recently proposed function (4) for calculating the coupling length in SI POFs with high NA (0.45 to 0.5$)$ is more accurate than Gloge's function (3) which substantionally underestimates the coupling length. The reason for this is that Gloge's function (3) takes into account neither the influence of NA on the coupling length, nor the width of the launch beam distribution. Finally, using our recently proposed method of calculating $L_{\mathrm{c}}$, one does not need to know the coefficient $A$ in (1). This is not the case when using Gloge's function.

\section{Conclusion}

We have tested the recently reported function (4) [9] for calculation of the coupling length (fiber length where the equilibrium mode distribution is achieved) in the case of SI POF. This function simplifies the determination of the coupling length by eliminating the need to numerically solve the power flow equation (or equivalent) repeatedly for every new case including every new geometric configuration (i.e. bending arrangement) of the fiber if strained fibers are considered as well [16]. We found that this function is more accurate for high-NA (0.45 to 0.5) SI POFs than Gloge's function (3) [7] that then substantionally underestimates the coupling length. The reason for this is that Gloge's function (3) takes into account neither the dependence of the coupling length on fiber's NA, nor the width of the launch beam distribution, which factors play more prominent role in high-NA fibers than in lower-NA fibers.

\section{Acknowledgments}

The work described in this paper was supported by a grant from City University of Hong Kong (Project
No. 7002313) and by a grant from Serbian Ministry of Science (Project No. 141037).

\section{References}

[1] J. Dugas, G. Maurel, Appl. Opt. 31, 5069 (1992).

[2] G. Jiang, R.F. Shi, F. Garito, IEEE Photon. Technol. Lett. 9, 1128 (1997).

[3] S. Zheng, X. Jin, X. Zhang, Microwave Opt. Technol. Lett. 48, 432 (2006).

[4] J. Mateo, M.A. Losada, I. Garcés, J. Zubía, Opt. Express 14, 9028 (2006).

[5] F. Breyer, M. Moerz, N. Hanik, L. Li, S. Randel, B. Spinnler, Eur. Trans. Telecomms. 18, 881 (2007).

[6] A.F. Garito, J. Wang, R. Gao, Science 281, 962 (1998).

[7] D. Gloge, Bell Syst. Tech. J. 52, 801 (1973).

[8] D. Gloge, Bell Syst. Tech. J. 51, 1767 (1972).

[9] A. Djordjevich, S. Savović, Opt. Eng. 47, 125001 (2008).

[10] W.A. Gambling, D.P. Payne, H. Matsumura, Appl. Opt. 14, 1538 (1975).

[11] J. Zubía, G. Durana, G. Aldabaldetreku, J. Arrue, M.A. Losada, M. Lopez-Higuera, J. Lightwave Technol. 21, 776 (2003).

[12] S. Savović, A. Djordjevich, Appl. Opt. 46, 1477 (2007).

[13] S. Savović, A. Djordjevich, Appl. Opt. 43, 5542 (2004).

[14] A. Djordjevich, S. Savović, J. Opt. Soc. Am. B 21, 1437 (2004).

[15] S. Savović, A. Djordjevich, Appl. Opt. 45, 6775 (2006).

[16] M.A. Losada, I. Garcés, J. Mateo, I. Salinas, J. Lou, J. Zubía, J. Lightwave Technol. 20, 1160 (2002). 